\title{
Identificación de acciones de salud en el Programa de Control de Síflis en gestantes, Cartagena de Indias, 2016
}

\author{
Identification of Health Actions in the Syphilis Control Program in Pregnant \\ Women, Cartagena de Indias, 2016
}

Elvisa Cárdenas Dávila ${ }^{1}$, Elías Alberto Bedoya Marrugo ${ }^{2}$, Yuri Arnold Domínguez ${ }^{3}$, Elin Manrique-Julio ${ }^{4}$

\section{Resumen}

Fundamento. La Sífilis Congénita es una infección causada por la diseminación hematógena del Treponema pallidum de la gestante infectada al feto. Se conoce que la transmisión materna puede ocurrir en cualquier momento del embarazo, por lo que el control de la enfermedad en la gestante es vital en la atención prenatal. Objetivo. Identificar el cumplimiento de acciones de salud comprendidas en el Programa de Control de Sífilis en gestantes de la ciudad de Cartagena de Indias, primer semestre del 2016. Materiales y métodos. Estudio observacional descriptivo transversal. Se evaluaron 781 gestantes inscritas en control prenatal de la ciudad, a través de las instituciones prestadoras de servicios de salud (IPS) y empresas promotoras de salud (EPS). Resultados. Concluyeron la gestación 358 (45.8\%) de las gestantes estudiadas. 25 (6.5\%) del total, no se realizaron la VDRL intraparto. Durante el primer trimestre del embarazo, el $99.6 \%$ de las evaluadas resultaron VDRL no reactivas (NR). Mientras que en el segundo trimestre fueron evaluadas, el $67.5 \%$ y el $57,4 \%$, respectivamente; resultando VDRL reactiva $(\mathrm{R})$ una $(0.1 \%)$ durante ambos semestres. Del segundo al tercer trimestre de la gestación no se incrementó el número de gestantes no evaluadas $(98 ; 12,5 \%)$ y $(63 ; 8,1 \%)$, sin embargo, sí se incrementó $(55 ; 19.8 \%)$ (269; $34.4 \%)$, respectivamente, el número de las gestantes que están a la espera del tiempo para realizar la prueba. No existió co-infección Sífilis/VIH/SIDA. Conclusiones. El control de la sífilis durante la atención prenatal fue deficiente dado por la alta tasa de no adherencia

\footnotetext{
1. Enfermera, Especialista en Salud Ocupacional, magister en Seguridad y Salud en el trabajo

Correo electrónico: benitacardenas2011@hotmail.com

ORCID: https://orcid.org/0000-0001-8087-3669

2. Administrador Servicios de Salud, Especialista en Salud ocupacional, Especialista en gestión de calidad y auditoría en Salud, Magister en Administración. Fundación Universitaria Tecnológico Comfenalco, Grupo CIPTEC.

Correo electrónico: eabedoya8@gmail.com

ORCID: http://orcid.org/0000-0002-2931-9600

3. Doctor en Medicina. Especialista de I y II grado en Higiene y Epidemiología. Universidad de Ciencias Médicas de La Habana. Investigador agregado del Instituto Nacional de Endocrinología, Cuba.

Correo electrónico: yuri.arnold@infomed.sld.cu

ORCID: https://orcid.org/0000-0003-4901-8386

4.Medica. Especialista en Salud Ocupacional, Magister en Toxicología. Universidad de Cartagena. Fundación BIOVIDA Correo electrónico: emanriquej@unicartagena.edu.co

ORCID: https://orcid.org/0000-0003-3092-9286
} 
al cumplimiento de asistencia a la consulta por la gestante, como la no realización de la prueba acorde al tiempo establecido por parte del equipo de salud.

Palabras claves: Embarazo; epidemiología; sífilis (fuente: DeCS BIREME).

\section{Abstract}

Basis. Congenital Syphilis is an infection caused by the hematogenous spread of Treponema pallidum from the pregnant woman infected to the fetus. At the time of pregnancy, control of the disease during pregnancy is vital in prenatal care. Objective. To identify the fulfillment of the health actions included in the Syphilis Control Program in pregnant women of the city of Cartagena de Indias, first half of 2016. Materials and methods. Cross-sectional descriptive observational study. A total of 781 prenatal control pregnant women were evaluated in the city, through the institutions that provide health services (PHI) and health promotion companies (PHE). Results. Gestation was completed in 358 $(45.8 \%)$ of the pregnant women studied. In $25(6.5 \%)$ of the total, intrapartum VDRL was not performed. During the first trimester of pregnancy $99.6 \%$ of the resulting nonreactive VDRL (NR) evaluations. While in the second trimester, they were evaluated, $67.5 \%$ and $57.4 \%$, respectively; resulting reactive VDRL (R) one $(0.1 \%)$ during both semesters trimesters. From the second to the third trimester of pregnancy, the number of pregnant women was not increased $(98,12.5 \%)$ and $(63,8,1 \%)$, however, if it increased $(55,19.8 \%)$ (269; $34.4 \%)$, respectively, pregnant women who are waiting for the time to perform the test. There is no co-infection Syphilis/HIV/ AIDS. Conclusions. The control of syphilis during prenatal care was deficient given the high rate of non-adherence in the compliance of the treatment in the pregnant woman, as their tests were not made according to the time established by the health team.

Keywords: Pregnancy; Epidemiology; Syphilis (source: DeCS BIREME).

\section{Introducción}

La sífilis es una enfermedad sistémica y crónica, causada por la espiroqueta Treponema pallidum subespecie pallidum, trasmitida principalmente por contacto sexual. Esta enfermedad es especialmente preocupante durante el embarazo debido al riesgo de in- fección transplacentaria en el feto [1] .Cada año se reportan de manera continua muertes fetales y mortalidad infantil temprana por sífilis [1,2]. La Organización Mundial de la Salud (OMS) estimó que cada año ocurren hasta 1,5 millones de casos de sífi- 
lis gestacional (SG) [3]; por lo cual, esta se considera un grave problema de salud pública. Debe mencionarse que América Latina y el Caribe (ALC) tiene una tasa de sífilis materna más alta que cualquier otra región, estimada por la OMS entre 1997 y 2003 en $3,9 \%$. Con dicha tasa se calcula que puede haber aproximadamente 459.108 casos de SG en la región de las Américas (exceptuando EE. UU. y Canadá), originando cada año de 164.222 a 344.331 casos de sífilis congénita $[4,5]$

La Organización Panamericana de la Salud (OPS) ha propuesto la meta de eliminar la sífilis congénita como problema de salud pública llevando la incidencia a 0,5 casos o menos, incluidos los mortinatos, por 1.000 nacidos. Para lograr esta meta, es necesario que se detecten y traten más de $95 \%$ de las gestantes infectadas, con lo que se logrará reducir la prevalencia de sífilis durante la gestación a menos de 1,0\% [5].

En Colombia, con los programas de vigilancia y control gestacional, la notificación de casos de SG y sífilis congénita (SC) han ido en aumento desde 1996. Sin embargo, aún existe subregistro en gran parte del territorio nacional. No obstante, se calculó que la proporción de incidencia de la SC ha pasado de 0,90 casos por 1000 nacidos vivos en 1998 a 2,43 casos en 2013 y la razón de prevalencia para SG de 1.3 casos por 1000 nacidos vivos en 2003 a 6,60 en 2013 [4,6]

La sífilis se puede dividir en varias etapas: sífilis primaria, secundaria, latente y tercia- ria. Las manifestaciones clínicas de la sífilis no están aparentemente alteradas por el embarazo y la transmisión vertical puede ocurrir en cualquier momento y etapa de la sífilis [7]. El riesgo de transmisión de la enfermedad se correlaciona con la extensión de la presencia de espiroquetas en la circulación sanguínea, por lo que la sífilis primaria y secundaria conllevan un mayor riesgo de transmisión que la sífilis latente y terciaria [8].

La SC es la complicación más devastadora de la SG y es causa de desenlaces adversos del embarazo como abortos, muertes fetales o neonatales (40-81\%), recién nacidos con bajo peso al nacer o con un cuadro clínico de SC (15-32\%) [5,8]

Las investigaciones señalan que los principales factores que determinan la infección fetal y sus resultados adversos son el estadio de la SG, así como el tiempo de exposición en el útero y por ello se considera prioritario detectar la infección materna precozmente de manera que se pueda realizar oportunamente el manejo que garantice la curación materna, prevenga la reinfección y elimine la infección congénita [5,6] La SG se diagnostica de manera similar a la población no embarazada. La OMS recomienda la realización de pruebas treponémicas y no treponémicas (FTA-ABS, RPR, VDRL ) para lograr establecer el diagnóstico de esta entidad $[8,9]$.

La detección sistemática y el tratamiento de las mujeres gestantes con sífilis se recono- 
ce como una intervención barata y factible para prevenir la sífilis gestacional, pero a pesar y de su naturaleza prevenible, la sífilis congénita sigue siendo común en muchas partes del mundo. Se cree que la mayoría de los casos de transmisión de sífilis durante el embarazo ocurren en el útero de forma transplacentaria, aunque es posible la transmisión durante el parto [12]. La infección en periodo de lactancia es poco frecuente, aunque se da en casos donde existen lesiones infecciosas en la mama [13].

Sin duda, la prueba prenatal de laboratorio para la sífilis juega un papel importante en el diagnóstico, y una diferencia significativa en el riesgo de tener un resultado adverso ya que la detección temprana permitirá un tratamiento temprano para la madre, lo cual reduce el riesgo SC [14]. Tras el diagnóstico de sífilis, las mujeres embarazadas deben realizarse un examen clínico y serológico mensual hasta el parto $y$, posteriormente, realizar un seguimiento como en pacientes no embarazadas y el tratamiento a la pareja sexual también es un aspecto importante para el manejo y la prevención de la enfermedad [15].

Las estrategias de salud pública para prevenir la SG y SG son similares a las que se enfocan en la sífilis y otras enfermedades de transmisión sexual en la población general, pero la detección prenatal de rutina es la principal línea de defensa contra la SC. El factor principal que explica la incapacidad para prevenir una infección congénita es la falta de atención prenatal. La probabilidad de buscar atención prenatal está fuertemente asociada con la edad, estado civil y socioeconómico, residencia rural y educación [16]. Se han determinado porcentaje de gestantes con sífilis en edades comprendidas entre 15 y 19 años, con especial énfasis en pacientes de raza negra, cuyo estado civil es casadas y con bajo nivel educativo, este último factor dificulta el acceso a los tratamientos para la enfermedad [17]. Para reducir la prevalencia de la SG y la SC es esencial que los profesionales de la salud y la comunidad tomen conciencia de la importancia del diagnóstico precoz y el tratamiento eficaz de las mujeres y sus parejas [18]. La eliminación de la SC como problema de salud pública solo será posible si se emprenden también intervenciones en los grupos vulnerables, como homosexuales, trabajadoras sexuales, consumidores de drogas y poblaciones móviles (migrantes, transhumantes, transportistas) [19]. Se requiere un esfuerzo para aumentar la conciencia sobre la extensión y la gravedad de la sífilis en el embarazo en todos los niveles de los servicios de salud, respaldados por un compromiso de política de estado.

\section{Metodología}

Se desarrolló un estudio descriptivo, que abarcó 781 gestantes evaluadas como asistentes a consulta externa en una IPS en la ciudad de Cartagena, durante el primer semestre de 2016. Se estudiaron clínicamente pacientes gestantes entre los 14 y 45 años de edad, consignando sus datos en la histo- 
ria clínica (datos sociodemográficos, afiliación al sistema, antecedentes personales y familiares). Se analizaron muestras de suero sanguíneo de las madres, donde se diagnosticaron mediante pruebas tamiz no treponémicas como la prueba de selección para sífilis Venereal Disease Research Laboratory (VDRL) aplicadas durante el primer, segundo y tercer trimestre del embarazo y prueba de VIH, sumado a la evaluación clínica, practicada a la madre gestante que permitieron la confirmación diagnóstica en relaciona a la sífilis. A partir de las evaluaciones realizadas, se recolectaron datos los cuales fueron tabulados y procesados mediante el diligenciamiento de cuadros en el programa excel, para proseguir con el manejo de las tablas y gráficos obtenidos principalmente en cifras porcentajes relacionadas.

\section{Resultados}

Se evaluaron 781 mujeres gestantes, con promedio de edad de 26 ańos (DS 6.03), donde la menor edad registrada fue 14 ańos y la máxima de 47 respectivamente; nivel socioeconómico, donde $623(80 \%)$ pertenecen al estrato 1; se hallaron 145 (19\%) pertenecientes al estrato 2 y $13(1 \%)$ al estrato 3; mientras que el $41 \%$ de las evaluadas son madres solteras y $51 \%$ viven en unión libre, $8 \%$ se han casado y un $1 \%$ son viudas (ver Tabla 1 ).

Tabla 1. Distribución sociodemográfica de las evaluadas.

\begin{tabular}{|l|l|l|}
\multicolumn{1}{c|}{ Total Evaluadas } & \multicolumn{2}{c|}{781} \\
\hline Estrato 1. & 623 & $80 \%$ \\
\hline Estrato 2. & 145 & $19 \%$ \\
\hline Estrato 3. & 13 & $2 \%$ \\
\hline Madre solteras & 321 & $41 \%$ \\
\hline Casadas & 59 & $8 \%$ \\
\hline Unión libre & 396 & $51 \%$ \\
\hline Viuda & 5 & $1 \%$ \\
\hline Primaria & 365 & $47 \%$ \\
\hline Secundaria & 359 & $46 \%$ \\
\hline técnico & 44 & $6 \%$ \\
\hline Profesional & 6 & $1 \%$ \\
\hline Otro & 7 & $1 \%$ \\
\hline
\end{tabular}

Fuente. Evaluación a gestantes del proyecto.

Del total, 365 (47\%) afirma haber estudiado hasta el nivel primario y otros 359 (46\%) hasta algún grado de secundaria; $44(6 \%)$ son técnicos en alguna disciplina mientras el $(1 \%)$ corresponde a profesionales y otro nivel educativo no especificado.
Se realizó un análisis del número de gestantes estudiadas que presentaron serología positiva y a la vez obtenida la causa se procede a revisar dicho comportamiento, tomando las características de las evaluadas de manera inicial, donde entre 781 evaluadas 715 
$(91,5 \%)$ se encontraban activas en alguna empresa promotora de servicios de salud y 66 (8.5\%) habían sido canceladas de algún ente de previsión. De los 715 activas en em- presa promotora de servicios de salud 713 (91.3\%) pertenecen al régimen contributivo, mientras que $2(0.3 \%)$ son del régimen subsidiado (ver Tabla 2 ).

Tabla 2. Características de gestantes evaluadas según régimen de afiliación.

\begin{tabular}{|l|l|l|}
\hline $\begin{array}{c}\text { Gestantes en cuenta de alto costo periodo 01/08/2015 - } \\
\text { 31/05/2016 }\end{array}$ & \multicolumn{2}{c|}{ Cantidad } \\
\hline Total gestantes inscritas en control prenatal en periodo & 781 & $\%$ \\
\hline Gestantes activas en asegurador & 715 & 91,5 \\
\hline Gestantes canceladas en asegurador & 66 & 8,5 \\
\hline Gestantes inscritas en control prenatal en contributivo & 713 & 91,3 \\
\hline Gestantes inscritas en control prenatal en subsidiado & 2 & 0,3 \\
\hline
\end{tabular}

Fuente. Encuestas epidemiológicas de la entidad promotora de salud.

Entre el grupo global de embarazadas objeto del estudio se ha determinado que $364(46.6 \%)$ pertenecen al grupo de edades comprendida entre los 25 y 35 años; mientras 334 de las evaluadas (42.8\%) están comprendidas entre los 15 y 25 ańos; un grupo de 79 embarazadas (10.1\%) se incluye en el grupo de 35 a 45 años; solo se encontraron 2 gestantes en los intervalos de las menores de 15 años y mayores de 45 años respectivamente (ver Gráfico 1).

Gráfico 1. Distribución de embarazadas organizadas por grupo etareo.

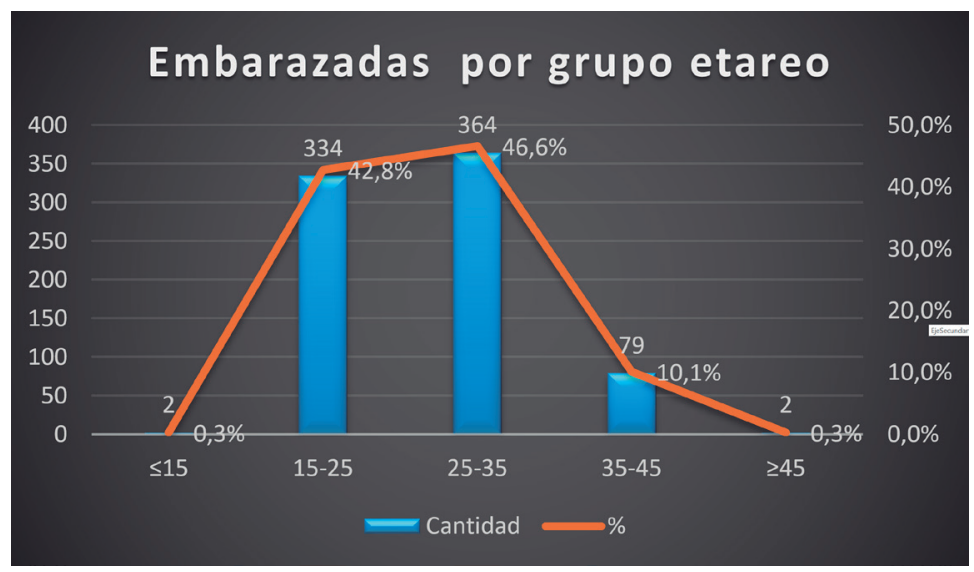

Fuente. Investigadores. 
De la muestra obtenida con 781 participantes, 66 de estas $(8.5 \%)$ se hallaron canceladas de los regímenes contributivo y subsidiado. Las 66 gestantes correspondientes al $8.5 \%$ que se registraban como canceladas de algún ente de previsión reciben igual atención por parte de las autoridades de salud distrital por parte del departamento ad- ministrativo distrital de salud (DADIS), lo cual evitará que estas queden excluidas del modelo de atención prenatal y parto, lo cual garantizará la atención prioritaria al estar embarazadas, incluidas en el programa especial de atención prenatal y cobertura por el Sistema de identificación de beneficiarias (SISBEN) a nivel nacional (Ver Gráfico 2).

Gráfico 2. Afiliación al Sistema de salud.

Afiliación de las gestantes a la fecha de estudio

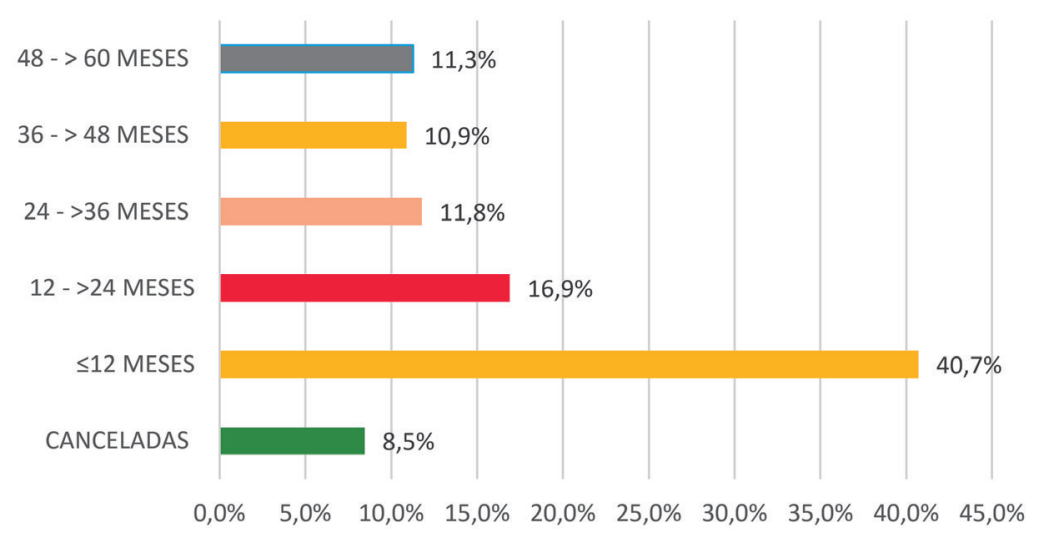

Fuente. Investigadores.

El promedio de meses de afiliación es de 45 (DS 31.5). Un grupo de 318 gestantes (40.7\%) presentaron afiliación a empresa promotora de servicios de salud durante un año o menos; mientras $132(16.9 \%)$ han estado afiliadas durante más de un año y menos de dos; 92 gestantes (11.8\%) están afiliadas entre 2 y tres ańos; 88 gestantes (11.3\%) se hallaron afiliadas durante más de 4 ańos y menos de 5. Sólo el porcentaje (10.9\%) agrupó a mujeres gestantes afiliadas entre tres y cuatro ańos (ver Gráfico 2). Lo anterior demuestra que la atención al servicio de salud bajo las diferentes mo- dalidades está cubierta, los impedimentos para acceder a las acciones de prevención y promoción para las gestantes se relacionan con el factor cultural, desinformación o no acudir a las instalaciones hospitalarias de primer y segundo nivel de atención, debido a que todas las gestantes evaluadas viven en el casco urbano de la ciudad de Cartagena. 383 gestantes llegaron al final del embarazo durante la investigación, 358 de estas (93.5\%), se evaluaron con VDRL intraparto y solo 25 de estas (6.5\%) no se realizó la mencionada prueba. Ninguna de las evaluadas en esta prueba resultó reactiva para 
sífilis. En cuanto a la evaluación por VDRL I, en el primer trimestre $99.6 \%$ de las evaluadas resultaron no reactivas para sífilis y solo tres del total no se realizó la mencionada prueba. En el segundo trimestre, 527 (27.5\%) fueron evaluadas, resultando reactiva para sífilis una gestante, un preocupante $12.5 \%$ no acudió a la evaluación y $19.8 \%$ está a la espera del tiempo (cumplir el se- gundo trimestre del embarazo) para realizar la prueba. También fueron evaluados con VDRL III 57.4\%, manteniéndose una gestante reactiva para sífilis y un $8.1 \%$ no acudió a realizarse el control respectivo, mientras otro $34 \%$ espera la fecha para realizar el procedimiento de control por VDRL III. De las 781 gestantes evaluadas ninguna dio positivo para VIH (ver Tabla 3).

Tabla 3. Resultados de pruebas para sífilis y VIH.

\begin{tabular}{|c|c|c|}
\hline $\begin{array}{c}\text { EVALUADOS POR VDRL } \\
\text { INTARPARTO }\end{array}$ & Cantidad & $\%$ \\
\hline CON VDRL INTRAPARTO & 358 & $93,5 \%$ \\
\hline SIN VDRL INTRAPARTO & 25 & $6,5 \%$ \\
\hline REACTIVO PARA SIFILIS & 0 & $0,0 \%$ \\
\hline NO REACTIVO PARA SIFILIS & 383 & $100,0 \%$ \\
\hline TOTAL & 383 & $100,0 \%$ \\
\hline EVALUADOS POR VDRL & Cantidad & $\%$ \\
\hline CON VDRL I & 778 & $99,6 \%$ \\
\hline REACTIVO PARA SIFILIS & 0 & $0,0 \%$ \\
\hline NO SE REALIZARON PRUEBAS & 3 & $0,4 \%$ \\
\hline A LA ESPERA DE PRUEBAS & 0 & $0,0 \%$ \\
\hline CON VDRL II & 527 & $67,5 \%$ \\
\hline REACTIVO PARA SIFILIS & 1 & $0,1 \%$ \\
\hline NO SE REALIZARON PRUEBAS & 98 & $12,5 \%$ \\
\hline A LA ESPERA DE PRUEBAS & 155 & $19,8 \%$ \\
\hline CON VDRL III & 448 & $57,4 \%$ \\
\hline REACTIVO PARA SIFILIS & 1 & $0,1 \%$ \\
\hline NO SE REALIZARON PRUEBAS & 63 & $8,1 \%$ \\
\hline A LA ESPERA DE PRUEBAS & 269 & $34,4 \%$ \\
\hline EVALUADOS POR VIH & Cantidad & $\%$ \\
\hline CON ELISA PARA VIH & 775 & $99,2 \%$ \\
\hline NO SE REALIZARON PRUEBAS & 6 & $0,8 \%$ \\
\hline POSITIVO PARA VIH & 0 & $0,0 \%$ \\
\hline TOTAL & 781 & $100,0 \%$ \\
\hline
\end{tabular}

Fuente. Investigadores. 


\section{Discusión}

Los resultados que se pueden visualizar en la tabla de distribución sociodemográfica indican que las personas objeto de estudio tienen la posibilidad de acceder con facilidad a la atención e información sobre la enfermedad, lo que configura una necesidad de ampliar los conceptos sobre cómo desarrollar una cobertura adecuada en salud que fundamente un mayor conocimiento sobre la enfermedad y su prevención; que a pesar de ser Colombia un país en vía de desarrollo puede decirse que está logrando un indicador favorable para evitar la propagación de infecciones y enfermedades de transmisión sexual, como la sífilis, contrariamente a lo indicado por el estudio de la OPS, en el cual se señala que los países no industrializados han de padecer de altos niveles de enfermedades transmisibles por su condición de subdesarrollo. También coincide con el estudio adelantado por Herrera y Rodríguez [17], quienes consideran que el bajo nivel educativo afecta decididamente a las mujeres frente al padecimiento de la sífilis, mientras se observa en los resultados de este estudio que todas las evaluadas ostentaban algún nivel educativo, lo cual les permite el acceso a la información de diferentes fuentes y además cuentan con edad promedio de 26 años y conforme estudios como el planteado por la anterior autora; es común encontrar pacientes en estos rangos de edad.

En este estudio no se abordó el aspecto racial para comprobar mayor afectación en- tendiendo que Colombia es un país multirracial y no hace distingos de color ni raza en sus servicios de salud, aunque en textos académicos aún se indica dicha característica como factor de riesgos a tener en cuenta.

En cuanto a la cobertura de los servicios de salud, esta es una fortaleza del sistema de salud colombiano, detectada en la anterior investigación debido a que la totalidad de las evaluadas tenían algún nivel de previsión social en salud, tanto en el régimen subsidiado como en el contributivo y a falta de estos dos, una tercera figura de aseguramiento, lo que conforme a la OMS permitirá la realización de las pruebas serológicas necesarias para lograr un diagnóstico que garantice el adecuado tratamiento de la enfermedad.

De acuerdo con la OMS, se estima que cada año ocurren hasta 1,5 millones de casos de sífilis gestacional. En el presente estudio solo se detectó un caso positivo de sífilis entre los 781 casos evaluados. Sin embargo, no deja de preocupar la deserción de gestantes en el proceso de control del embarazo, lo cual es un factor igualmente peligroso si de eventos recurrentes se trata, donde al generarse SG y por ende se producirá SC que al no ser diagnosticada ni tratada puede producir todas las complicaciones de salud ya mencionadas e incluso la muerte fetal, por los cual no deja de ser un problema de salud pública bastante considerable.

En este estudio no se abordó la temática de afinidades sexuales; sin embargo, las evalua- 
das tuvieron a bien manifestar su heterogeneidad sexual, aunque no deja de ser una característica importante a resaltar como el acercamiento que estas puedan tener con grupos de comunidad homosexual, consumidores de drogas y las poblaciones móviles (migrantes, transhumantes, transportistas) mencionados ampliamente en varias investigaciones [19-21], sobre sífilis materna y sífilis congénita.

\section{Conclusión}

Las mujeres gestantes infectadas por sífilis pueden transmitir la infección al feto, provocándole sífilis congénita, que conlleva efectos adversos graves para el embarazo en hasta el $80 \%$ de los casos. Por ello, la asistencia al control prenatal es importante, y es pilar fundamental en la detección eficaz de alteraciones en el embarazo. Debido a que, de no tratar adecuadamente la sífilis, la embarazada puede sufrir afecciones serias en su gestación tales como aborto espontáneo, muerte fetal, nacimiento prematuro y Sífilis Congénita. Debido a la conexión existente entre la sífilis congénita y el virus de la inmunodeficiencia humana (VIH) las pacientes halladas positivas para sífilis también son susceptibles para la prueba de VIH. La detección temprana de ambas afecciones se obtiene con un análisis de sangre; si resulta reactivo para sífilis, un sencillo tratamiento a base de penicilina permite curar tanto a la mamá como al bebé. Cabe resaltar que la sífilis congénita es una enfermedad evitable con tratamiento específico, eficaz y precoz, además es de bajo costo y se encuentra disponible en todos los servicios de salud del primer nivel de atención. Prevenir la transmisión materno-infantil de la sífilis cuesta un poco más de un dólar, la mayor peligrosidad en este caso es la no asistencia al servicio de salud para informarse a tiempo evitando el contagio y las complicaciones que este trae para la madre y el bebé.

\section{Referencias}

1. A. A. Wahab, U. K. Ali, and M. Mohammad, "Syphilis in pregnancy," Pakistan J. Med. Sci., vol. 31, no. 1, pp. 2014-2016, 2014.

2. M. Magalhães, L. Basto, A. Luísa, M. Eulália, and A. Paulo, "Syphilis in Pregnancy and Congenital Syphilis : Reality in a Portuguese Central University Hospital," Rev. Bras. Gineoobstetricia, vol. 39, no. 6, 2017.

3. World Health Organization, Infecciones de Transmision Sexual 2016-2021. Geneva, Switzerland., 2016, pp. 5-64.

4. I. N. de Salud, Protocolo de Vigilancia en Salud Pública: Sifilis gestacional y Sifilis congenita. Colombia, 2015, pp. 2-40.

5. OPS, Guía clinica para la eliminación de la transmisión materno infantil del VIH y de la sifilis congénita en América Latina y el Caribe. USA, Washington D.C., 2009, pp. 1-100.

6. M. de S. de Colombia, Guía de práctica clínica ( GPC) basada en la evidencia para la atención integral de la sifilis gestacional y congénita. Colombia, 2014, pp. 3-25.

7. M. De Santis et al., "Syphilis Infection during Pregnancy : Fetal Risks and Clinical Management," Infect. Dis. Obstet. Gynecol., vol. 2012, pp. 3-5, 2012. 
8. J. E. Stoltey, S. E. Cohen, S. Francisco, and S. Francisco, "Syphilis transmission : a review of the current evidence," Sex Heal., vol. 12, no. 2, pp. 103$109,2018$.

9. R. E. Plotzker, R. D. Murphy, and J. E. Stoltey, "Congenital Syphilis Prevention: Strategies , Evidence, and Future Directions," Sex. Transm. Dis., vol. 45, no. 9, pp. 29-37, 2018.

10. M. de S. de Colombia, Plan de Intensificacion para la Eliminacion de la Sifilis Congenita. Colombia, 2016, pp. 6-10.

11. R. Creasy, Robert K. Resnik, Creasy and Resnik's Maternal-Fetal Medicine, 7th ed. Philadelphia, 2014.

12. J. S. Slutsker, R. R. Hennessy, and J. A. Schillinger, "Factors Contributing to Congenital Syphilis Cases — New York City , 2010 - 2016," MMWR Morb Mortal Wkly Report., vol. 67, no. 39, pp. 10881093, 2018.

13. W. J. Ledger, "Syphilis in pregnancy Review," $B r$. Med. J., vol. 76, no. 2, pp. 73-79, 2000.

14. S. J. Hawkes, G. B. Gomez, and N. Broutet, "Early Antenatal Care: Does It Make a Difference to Outcomes of Pregnancy Associated with Syphilis? A Systematic Review and Meta-Analysis," PLoS One, vol. 8, no. 2, 2013 .

15. W. H. Organization, Syphilis screening and treatment for pregnant women. Geneva, Switzerland, 2017.

16. M. L. Kamb et al., "A Road Map for the Global Elimination of Congenital Syphilis," Obs. Gynecol Int., vol. 2010, pp. 1-6, 2010.

17. L. . Herrera,Vivian. Rodríguez, "Incidencia de la sífilis durante el embarazo," Rev. Cuba. Med. Gen. Integr., vol. 13, no. 4, pp. 352-358, 1997.

18. C. Padovani, R. R. De Oliveira, and S. M. Pelloso, "Syphilis in during pregnancy: association of maternal and perinatal characteristics in a region of southern Brazil 1,” 2018.

19. Pereira Palacio, J., Gaviria Mejía, L., Zea-Castrillón, S., Jaramillo Arbleáez, P., \& Bedoya, A. (2018). Des- cripción de pruebas moleculares en el diagnóstico del virus Zika en el periodo 2008-Febrero 2018. Revisión sistemática. NOVA, 16(30), 81-93. https:// doi.org/10.22490/24629448.2838

20. Pinilla B., G., Chavarro, B., Moreno, N., Navarrete, J., \& Muñoz, L. (2015). Determinación de los genes, 16S ADNr, polA, y TpN47, en la detección de Treponema pallidum subsp. pallidum para el diagnóstico de sífilis congénita. NOVA, 13(24), 17-26. https://doi.org/10.22490/24629448.1713

21. F. ValderramaI,Julia. Zacarías, "Maternal syphilis and congenital syphilis in Latin America: big problem, simple solution," Rev. Panam. salud publica, vol. 16, no. 4, pp. 211-217, 2004. 